\title{
Stuck between a rock and a hard place: ILD + NSCLC $=$ LIM
}

\author{
Waël C. Hanna, MDCM, MBA, FRCSC
}

From the Division of Thoracic Surgery, McMaster University, Hamilton, Ontario, Canada.

Disclosure: Author has nothing to disclose with regard to commercial support.

Received for publication May 8, 2017; accepted for publication May 11, 2017; available ahead of print June 16, 2017.

Address for reprints: Waël C. Hanna, MDCM, MBA, FRCSC, St. Joseph's Healthcare Hamilton, 50 Charlton Ave E, Juravinski Tower, Suite T2105 F, Hamilton, Ontario, Canada L8N4A6 (E-mail: hannaw@mcmaster.ca).

J Thorac Cardiovasc Surg 2017;154:1074

$0022-5223 / \$ 36.00$

Copyright (c) 2017 by The American Association for Thoracic Surgery

http://dx.doi.org/10.1016/j.jtcvs.2017.05.031

In the complicated world of interstitial lung disease (ILD), in which a plethora of acronyms, such as UIP, NSIP, IP, and IPF, abound, the surgeon's decision making should be simple and based solely on one acronym: LIM-less is more.

When patients with ILD are diagnosed with early-stage non-small cell lung cancer (NSCLC), a dilemma sometimes arises. On one hand, surgical resection with lobectomy and lymphadenectomy is thought to confer the best survival, but on the other hand, lobectomy is also associated with further reductions in lung function, increased risk of exacerbation, and increased short-term mortality. In patients who cannot physiologically tolerate lobectomy, most surgeons stick to the LIM principle and typically recommend limited resection; however, in patients who can tolerate lobectomy, should the LIM principle still apply at the expense of suboptimal cancer control?

In this issue of the Journal, Tsutani and colleagues ${ }^{1}$ attempted to answer this question by comparing short- and medium-term outcomes of 2 cohorts of patients with ILD and stage I NSCLC who underwent either limited resection or lobectomy. They found that 90-day mortality did not differ between the 2 groups, but that the incidence of high-grade complications was substantially higher, although not statistically significantly so, in the lobectomy cohort. They also found that overall survival and cancerspecific survival at 36 months also did not differ between the 2 cohorts. Interestingly, they conclude that sublobar resection may be a suitable alternative to lobectomy.

I find their conclusion interesting, because the reverse could have just as easily been true. In the absence of any differences in mortality, complications, or oncologic survival, one could conclude that lobectomy is a suitable operation in this selected population, and that the LIM principle should not apply. However, a closer look at this study reveals a retrospective analysis that is severely limited by selection

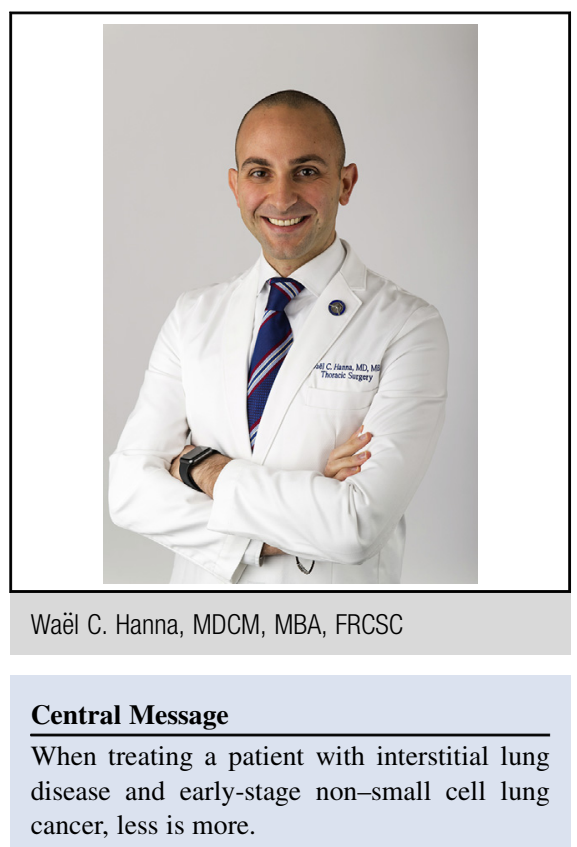

See Article page 1089.

bias, for which the authors were not able to adjust. The Cox proportional hazards model fails to include oncologically important variables, such as tumor size and lymph node status. More importantly, the study is also severely underpowered to justify a noninferiority equivalence conclusion. In the absence of rigorous data, the authors are justified in erring on the side of the prevailing principle of LIM and proposing sublobar resection as an adequate operation in this population.

When treating a patient with ILD and early-stage NSCLC, surgeons should not feel stuck between a rock and a hard place. This is especially true at a time when lobectomy is being challenged as the standard of care for stage I NSCLC, even in patients with normal lung function. When in doubt, surgeons should remember the only acronym that matters for interstitial lung disease: LIM.

\section{Reference}

1. Tsutani Y, Mimura T, Kai Y, Ito M, Misumi K, Miyata Y, et al. Outcomes after lobar versus sublobar resection for clinical stage I non-small cell lung cancer in patients with interstitial lung disease. J Thorac Cardiovasc Surg. 2017;154: 1089-96.e1. 